\title{
A Gas Leak Rate Measurement System for the ATLAS MUON BIS-Monitored Drift Tubes
}

\author{
T. Alexopoulos, R. Avramidou, M. Dris, T.A. Filippas, E.N. Gazis, \\ E.C. Katsoufis, S. Maltezos, P. Savva, G. Stavropoulos, G. Tsipolitis
}

\author{
NTU Athens - Physics Department \\ Faculty of Applied Sciences \\ Zografou Campus GR-157 80 \\ Athens, Greece
}

\begin{abstract}
A low-cost, reliable and precise system developed for the gas leak rate measurement of the BIS-Monitored Drift Tubes (MDTs) for the ATLAS Muon Spectrometer is presented. In order to meet the BIS-MDT mass production rate, a total number of 100 tubes are tested simultaneously in this setup. The pressure drop of each one of the MDT is measured, within a typical time interval of 48 hours, via a differential manometer comparing with the pressure of a gas tight reference tube. The precision of the method implemented is based on the system temperature homogeneity, with accuracy of $\Delta \mathrm{T}=0.3{ }^{\circ} \mathrm{C}$. For this reason, two thermally isolated boxes are used testing 50 tubes each of them, to achieve a high degree of temperature uniformity and stability. After measuring several thousands of the MDTs, the developed system is confirmed to be appropriate within the specifications for testing the MDTs during the mass production.
\end{abstract}




\section{Introduction}

A system of 128 Barrel Inner Small (BIS) chambers, consisted of almost 30000 aluminum Monitored Drift Tubes (MDT) with a $167 \mathrm{~cm}$ length, is going to be built in Greece for the ATLAS Muon Spectrometer [1].

The gas leak rate measurement of the BIS-MDTs is part of the quality assurance and quality control (QA_QC) procedure, in addition to the anode wire tension, the leakage current under high voltage and the anode wire displacement measurements. The QA_QC procedure is part of the cooperation, for the construction of the BIS chambers in Greece, among three Greek institutions, the National Technical University of Athens (NTUA), the University of Athens (UoA) and the Aristotle University of Thessaloniki (AUTH). The construction of the tubes takes place at the UoA [2], the QA_QC at the NTUA [3] and the final MDTs assembly of the BIS-chamber at the AUTH [4].

The gas leak rate measurement of the MDTs, which are filled with a mixture of Ar: $\mathrm{CO}_{2}$ (93:7) at an absolute pressure of 3 bars, is a crucial detector performance parameter. The developed experimental setup measures the average gas leak rate, of 2 groups of 50 MDTs simultaneously in order to meet the BIS-MDT mass production rate. This technique scans the gas leak rate of each MDT, testing the end-plugs and the cylindrical surface. The method, which has been implemented, measures the pressure drop via a differential manometer [5], between a gas tight reference tube and each one of the under test MTDs, in a time interval of 48 hours. Some preliminary tests and results have been presented previously $[3,6]$.

The gas leak rate measurement method of the MDTs is sensitive to the temperature stability and uniformity along the measured tubes and the reference tube in the setup. For this reason, attention is given to avoid significant temperature effects. Thus specific Temperature Stabilization Boxes (TSB) have been designed and constructed to enclose the tubes under testing.

Reference tubes are used in order to compensate the residual temperature effects in the data analysis. The overall estimated error of the gas leak rate is $\sim 6 \%$ of the maximum allowed value $\left(10^{-8} \mathrm{bar} \cdot \mathrm{l} / \mathrm{s}\right)$ for the selected time interval of the measurement. For absolute calibration purposes monitored gas micro-flows through capillary tubes with certain accurate size dimension (10 $\mu m$ diameter) are used.

The temperature monitoring and the differential manometer data taking are computer controlled, while the overall gas leak rate test procedure provides a data file of the gas leak rate and the id per MDT, in a semi-automatic way.

In the following, the method and the experimental setup are presented in section 2 , the data analysis for the gas leak rate determination, the estimated precision and the necessary corrections in section 3 . In section 4 , the evaluation measurements are analyzed, while in section 5 the conclusions and the future work are discussed.

\section{The gas leak rate test setup}

\subsection{System configuration}

The 100 MDTs to be tested, in every measurement, are placed inside two similar TSBs, which do not use any active system for heating. These boxes have been 
specifically designed and constructed for this purpose. A schematic diagram is shown figure 1 . The box A is made entirely from wood, while the box B has a metallic frame with wooden walls. The walls of both boxes are covered internally by a thermal isolation material of $10 \mathrm{~mm}$ thickness and thermal conductivity $\lambda=0.038 \mathrm{~W} / \mathrm{m} \mathrm{K}$ (DIN 52612). The thermal loss coefficient of the isolation material is $\mathrm{k}=\lambda / \mathrm{d}=3.8 \mathrm{~W} / \mathrm{m}^{2} \mathrm{~K}$, while the overall effective $\mathrm{k}$ of the walls is estimated to be $2.9 \mathrm{~W} / \mathrm{m}^{2} \mathrm{~K}$. The setup is located in a room where the temperature is stabilized within $\pm 0.5{ }^{\circ} \mathrm{C}$. The short period variations of the room temperature are smoothed well enough inside each box because of the achieved high value of the "time constant".

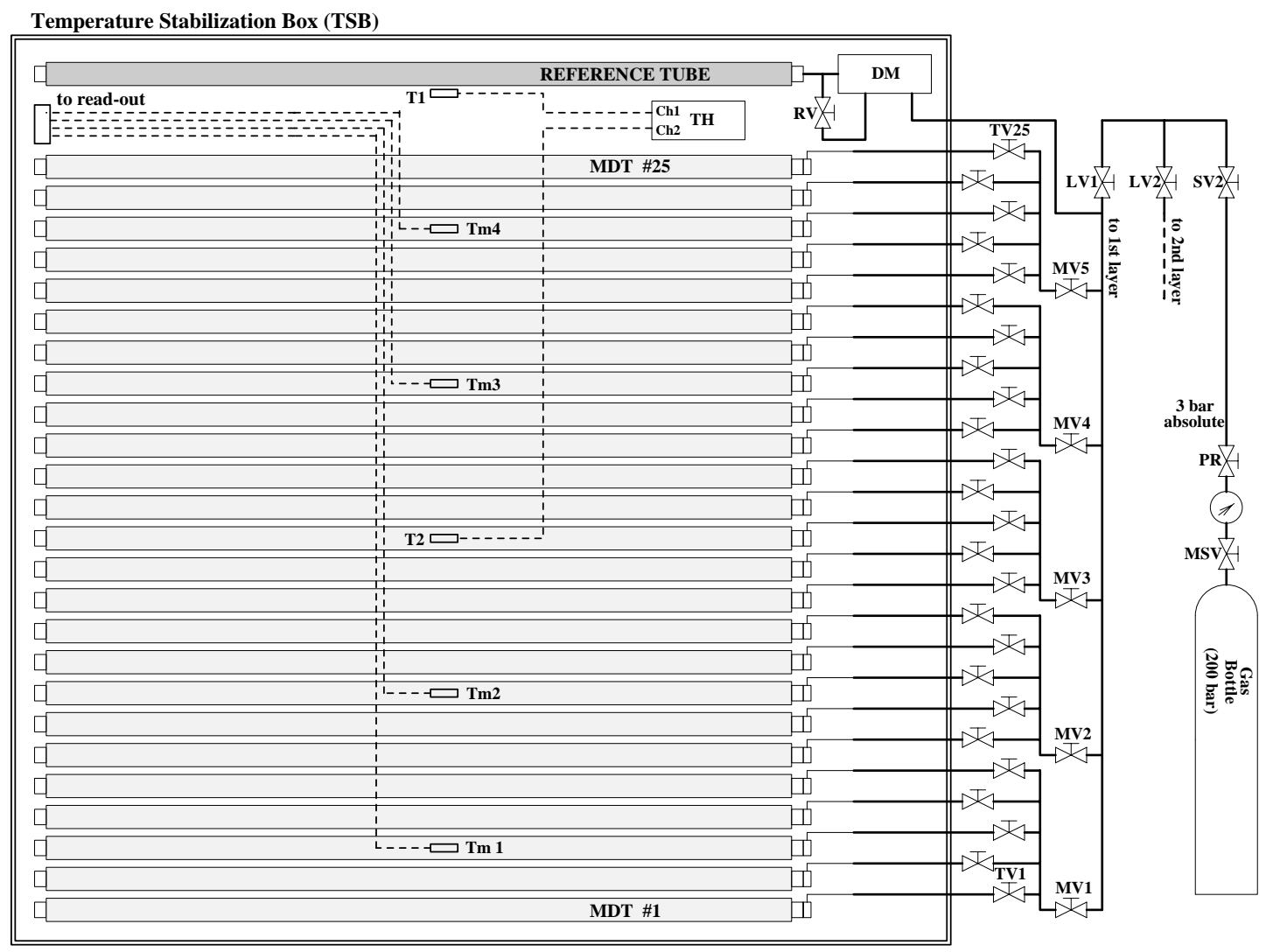

Figure 1. A schematic diagram of the experimental setup showing one layer of 25 tubes (MDTs); differential manometer (DM); dual thermometer (DT); tube valves (TVi); manifold valves $(M V i)$; line valves (LVi); reference valve $(R V)$; main supply valve $(M S V)$; pressure reducer (PR); supply valve (SV2), i-temperature sensor channel (Chi).

Test measurements have shown that the temperature variation for a time interval of $1 \mathrm{~h}$ is reduced by a factor of 5 inside the box. A general photo view of the gas leak rate measurement system is presented in figure 2.

A dual channel thermometer (DT) ${ }^{1}$, with Pt100 thermocouples and a precision of $0.01{ }^{\circ} \mathrm{C}$ for the temperature measurement, is used to record the temperature in the reference tube (sensor T1) and in the middle of the MDTs layer (sensor T2) as well.

An additional thermometer ${ }^{2}$ with an accuracy of $0.03{ }^{\circ} \mathrm{C}$ is used to monitor the room temperature. Four Pt100 temperature sensors $\mathrm{Ch} 1, \mathrm{Ch} 2, \mathrm{Ch} 3$ and $\mathrm{Ch} 4$, per each

\footnotetext{
${ }^{1}$ Bioblock Scientific, SWITZERLAND

${ }^{2}$ Vaisala Oyj, FINLAND
} 
box, are used to record the temperature of the tubes near the walls of that, since some fringe temperature variations have been observed at the walls of the boxes. The read-out electronics are based on the LMB card [5], which uses the CANbus. The monitoring of the temperature is performed with BridgeVIEW ${ }^{3}$. More technical information about this temperature monitoring system will be given in a future ATLAS-Note [6].

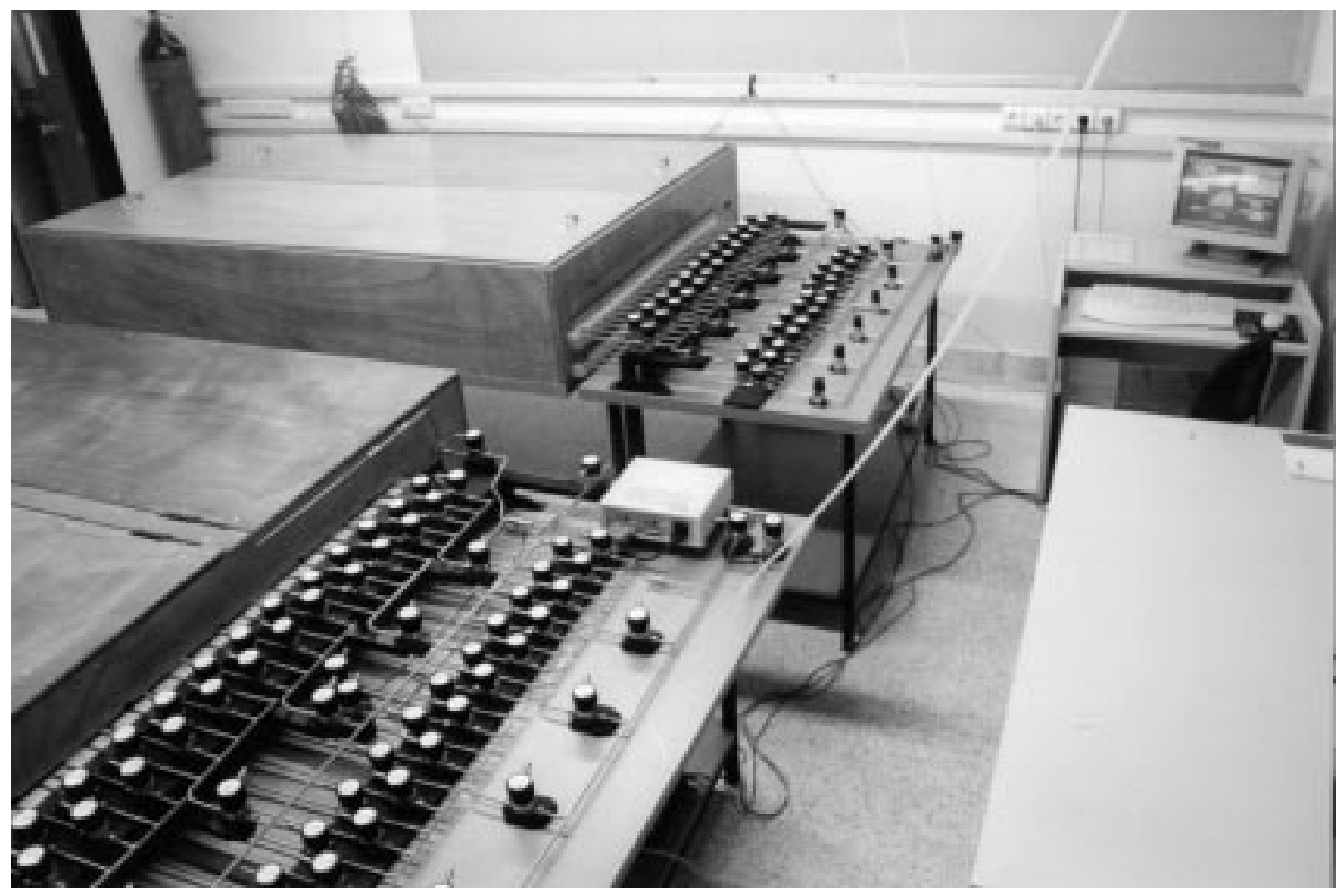

Figure 2. A view of the two Temperature Stabilization Boxes, (TSB) and the respective valve manifold complex. Each valve socket is connected with the corresponding tube (MDT) inside the box via a flexible thinner stainless steel tube.

For the gas leak rate measurement, 50 tubes are placed in each TSB arranged in two layers of 25 tubes (lower and upper). Bundles of 5 MDTs are connected to a corresponding manifold, which is also connected to the main gas supply system. One differential manometer $(\mathrm{DM})^{4}$ per each layer of 25 MDTs is used, thus two DMs are used in each TSB, with an individual reference line connected to each reference. The measuring line of the DM can be connected directly to MDT under test and sequentially to the each one of the rest MDTs using the corresponding valve. The DM has a full range from 0 to 5 PSID (344.75 mbar), giving an analog voltage output in the range 0-5 $V$.

The MDTs are filled with the detector operating gas, $\mathrm{Ar}: \mathrm{CO}_{2}$ (93:7), supplied from a pre-mixed bottle via a pressure reducer. The applied pressure is 2 bars relative to the atmospheric air ( 3 bars absolute). As a result, the actual mixture for the gas leak rate test contains finally $2 / 3$ parts of the above gas mixture and $1 / 3$ part of the atmospheric air. Each MDT is connected to its corresponding pipe via its individual valve of

\footnotetext{
${ }^{3}$ National Instruments, USA

${ }^{4}$ Sensotec, USA, type FP2000/mod FDD1
} 
diaphragm type, HP-200-IM ${ }^{5}$. These valves have an extremely low gas leakage between the inlet, outlet and their body, which is less than $10^{-12} \mathrm{bar} \cdot \mathrm{l} / \mathrm{s}$ with He gas, as it is specified by the manufacturer.

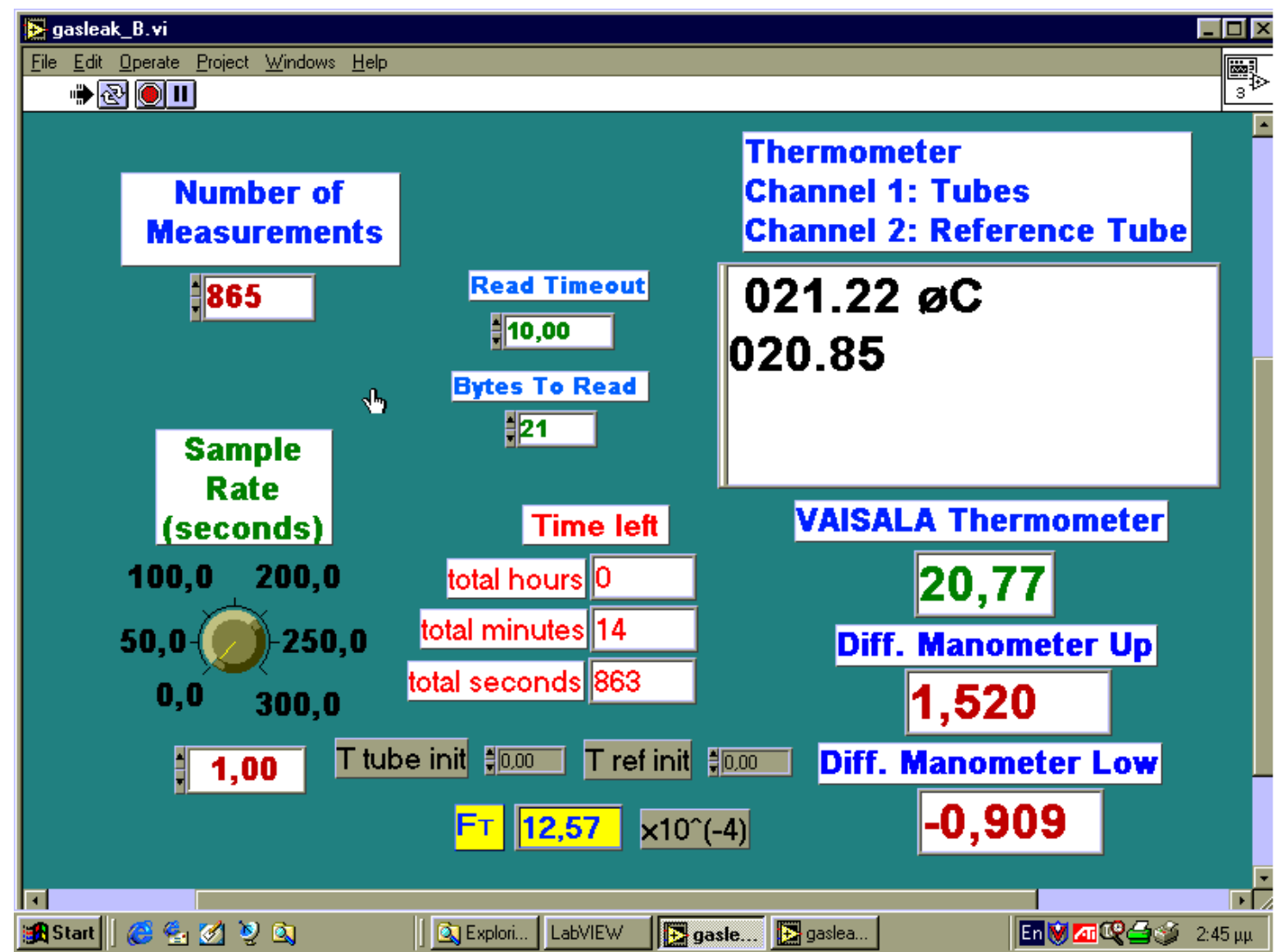

Figure 3. The operating panel of the LabVIEW for the differential pressure measurement in the TSB via the DM (Up and Low levels of the MDTs) and the temperature measurement via the DT (chan.1: MDTs, chan.2: Reference Tube). In addition, the total number of measurements and the sampling period can be selected, according to the total time interval.

The gas leak rate measurement consists of the following three main steps described below:

- Tube mounting and gas filling (ST $\left.\mathbf{S T}_{1}\right)$ : The MDTs to be tested are together with the reference tube and are filled with the gas mixture under the absolute pressure of 3 bars. The system remains in this state for at least $12 \mathrm{~h}$, in continuous gas supply and pressure plus temperature stabilization for reduction of any transient phenomena of gas leaking inside the micro-cavities or structure micro-packets of the end-plugs material made of Noryl. [7]

- Starting the gas leak test $\left(\mathbf{S T}_{2}\right)$ : The valve connected to the reference tube is closed and all the initial temperatures are recorded. Then, all the valves of the MDTs are closed in sequence, and the starting time is recorded.

\footnotetext{
${ }^{5}$ Air Liquide, Gaz Industrieles Scientifiques, FRANCE
} 
- Measurement of the pressure drop $\left(\mathbf{S T}_{\mathbf{3}}\right)$ : After an elapsed time interval of at least $48 \mathrm{~h}$, the differential pressures in the manifold and in each MDT are recorded before and after opening the tube valves.

\subsection{On-line monitoring}

The experimental procedure of the gas leak rate measurement is controlled via the LabVIEW $^{6}$. The parameters recorded during the measurement are the temperatures from the pt-100 sensors and the differential pressure from the DM. A 12-bit/16-channel ADC-PCI card $^{6}$, is used for the digitization of the incoming analog data. The main two temperature values are digitized by the dual channel thermometer (DT) and transferred directly to the computer via the RS-232 port. Thus, the high accuracy of the dual channel thermometer is exploited, avoiding possible quantization errors from the A/D conversion.

In the control panel presented in figure 3 , the total number of measurements and the sampling period can be selected, according to the total time interval. The evolution of the gas leak rate measurement (step $\mathrm{ST}_{3}$ ) is monitored and reflected in the factor, $F_{T}$, which indicates the variation of the temperature conditions (see the data analysis section). The appropriate measuring time is selected under the condition that the $F_{T}$ is maintained within the allowed accepted limits $\left( \pm 3 \times 10^{-4}\right)$ and preferably close to zero, in order to minimize the systematic error.

\section{Data analysis}

\subsection{The gas leak rate determination}

In order to obtain the time depended gas leak rate, $L(t)$, of a MDT with a fixed volume $V$, (having an active length $l=165 \mathrm{~mm}$ and an internal diameter $d=2.96 \mathrm{~mm}$ ), we apply the mass flow of the gas expressed by the rate, $d n / d t$, calculated from the ideal gas law equation, $P V=n R T$ :

$$
L(t)=-R T \cdot \frac{d n}{d t}=-\frac{d P}{d t} V
$$

assuming that the system has a constant temperature. The minus sign is used to express the gas leak. According to eq. 1, for practical reasons the gas leak rate, $L(t)$, is expressed the gas leak rate $L(t)$ in units of pressure times volume per unit time.

It is considered a state transition, assuming constant temperature, from an initial state, $S^{o}\left(P_{o t}, n_{o}, T_{o}\right)$, to a final one, $S\left(P_{t}, n, T_{o}\right)$. The cause of this transition is the pressure change $P_{o t}-P_{a}$, after a certain time interval, where $P_{a}$ is the atmospheric pressure.

The gas leak rate depends on the difference $P(t)-P_{a}$ and also on the so called "conductance", $C$, of the system, that is:

\footnotetext{
${ }^{6}$ National Instruments, USA
} 


$$
L(t)=C\left[P(t)-P_{a}\right]
$$

substituting into the eq. 1 and solving the equation we get:

$$
P(t)=P_{a}+\left(P_{o t}-P_{a}\right) e^{-\frac{t}{T}}
$$

where, $T_{s}=V / C$ is the "time constant" of the system. The derivative of the pressure with respect to time gives:

$$
\frac{d P}{d t}=-\frac{\left(P_{o t}-P_{a}\right)}{T_{s}} e^{-\frac{t}{T}}
$$

for $t \cong 0$ the gas leak rate is expected to be close to the nominal one, thus the value $T_{s}$ obtained from eq. 4 is about $2.2 \times 10^{8} \mathrm{~s}$ (or 2574 days), which is much grater than the time interval, $\Delta t=172800 \mathrm{~s}(=48 \mathrm{~h})$, used in our measurement. Consequently, the pressure derivative could be considered as constant fo rthe interval of the measurement:

$$
d P / d t=-\left(P_{o t}-P_{a}\right) / T_{s}=C\left(P_{o t}-P_{a}\right) / V
$$

therefore, the average gas leak rate $L$ within time $\Delta t$, as calculated below, essentially expresses the time derivative of the pressure $d P / d t$ :

$$
L=-\frac{1}{\Delta t} \cdot \int_{0}^{\Delta t} L(t) d t=-\frac{V}{\Delta t} \cdot \int_{0}^{\Delta t} \frac{d P(t)}{d t} d t=\frac{\left(P_{o t}-P_{t}\right) V}{\Delta t}
$$

during the time interval $\Delta t$, the atmospheric pressure $P_{a}$, fluctuates due to the temperature changes caused upon various reasons, and could affect the conductance and consequently the calculated average $L$. However, this effect is estimated to be negligible (less than $0.3 \%$ ).

The gas leak rate, $L(t)$, of our system is obtained from the total pressure drop, using the differential pressure measured values instead of the absolute ones. In this calculation, the pressure drop of the reference tube is omitted because it is, obviously, considered negligible compared to that of the MDT under test, $\left(L<1 \times 10^{-9} \mathrm{bar} \cdot \mathrm{l} / \mathrm{s}\right)$, this is due to the special construction of the reference tube, having been confirmed experimentally. The gas leak rate, $L(t)$, for a MDT of a volume $\mathrm{V}$ within a time interval $\Delta \mathrm{t}$, is given by the relation:

$$
L=\frac{\left(\Delta P-\Delta P_{O S}\right) \cdot V}{\Delta t}
$$


where $\Delta P$, is the differential pressure of the MDT tube with regard to a reference pressure (reference tube) and $\Delta P_{o s}$ is the differential pressure of the DM obtained when its two inputs are connected together, having exactly the same pressures, called "pressure offset".

\subsection{The differential pressure correction}

The measured differential pressure for each MDT, obtained by the differential manometer (DM), is influenced by the temperature condition variation of the MDTs between the time start and stop during the test procedure. The final differential pressure $\Delta P^{\prime}$ measured by the $\mathrm{DM}$, as a result from the MDTs gas leak rate and its temperature variation effect, is described by:

$$
\Delta P^{\prime}=\left(P_{o r} \frac{T_{r}}{T_{o r}}-P_{o t} \frac{n}{n_{o}} \frac{T_{t}}{T_{o t}}\right)
$$

where $P_{o t}$, is the initial absolute pressure of the gas in the MDTs and $P_{o r}$ respectively the initial pressure of the reference tube. Similarly, $T_{o t}, T_{o r}$ are the initial and $T_{t}, T_{r}$ the final temperature of the MDTs under test and the reference tube respectively. Also, $n_{o}$ and $n$ are the number of molecules inside the MDTs at the initial and final conditions, respectively. In the eq. $8, P_{t}=P_{o t} n / n_{o}$ using the final pressure, $P_{t}$, of the tube and the final pressure of the reference tube, $P_{r}$, which is practically identical with the initial one, $P_{o r}$, due to the negligible leakage of the reference tube:

$$
\Delta P^{\prime}=\left(P_{r} \frac{T_{r}}{T_{\text {or }}}-P_{t} \frac{T_{t}}{T_{\text {ot }}}\right)=\frac{T_{t}}{T_{\text {ot }}}\left(P_{r} \cdot R_{T}-P_{t}\right)
$$

the quantity $R_{T}$ indicates the relative degree of variation of the temperature conditions between the MDT and the reference tube and it is defined as:

$$
R_{T}=\frac{\left(\begin{array}{l}
T_{o t} \\
T_{t}
\end{array}\right)}{\left(\frac{T_{o r}}{T_{r}}\right)}=\frac{T_{o t}}{T_{t}} \cdot \frac{T_{r}}{T_{o r}}
$$

In order to obtain a more convenient expression, we introduce in our measurements the quantity $F_{T}=1-R_{T}$, which is called Temperature Influence Factor (TIF). This factor represents the relative variation of the current conditions with respect to the initial ones.

In particular, we assume $\delta T_{\text {tr }}$ the difference of the temperature variation between the MDT under test and the reference tube, respectively: 


$$
\delta T_{\mathrm{tr}}=\delta T_{t}-\delta T_{r}=\left(T_{t}-T_{o t}\right)-\left(T_{r}-T_{o r}\right)
$$

considering that $\delta T_{t} \cong \delta T_{r}<T_{t}$ and $T_{t} \cong T_{r}=T$, which are indeed valid, it can be shown that $F_{T} \cong \delta T_{t r} / T$. Therefore, the eq. 9 can be written:

$$
\Delta P^{\prime}=\frac{T_{t}}{T_{o t}}\left[P_{r} \cdot\left(1-F_{T}\right)-P_{t}\right]
$$

arranging the terms it is obtained:

$$
\Delta P^{\prime}=\frac{T_{t}}{T_{o t}}\left(P_{r}-P_{t}-P_{r} F_{T}\right)
$$

where, the essential pressure difference $\Delta P=P_{r^{-}} P_{t}$, can be calculated as follows:

$$
\Delta P=\frac{T_{o t}}{T_{t}} \Delta P^{\prime}+P_{r} F_{T}
$$

the fraction $T_{o t} / T_{t}$ is approximately equal to one, within an error, which is less than $3 \times 10^{-4}$. Therefore, the eq. 14 becomes:

$$
\Delta P \cong \Delta P^{\prime}+P_{r} F_{T}
$$

in the last equation, the term $P_{r} F_{T}$ corrects the measured quantity for obtaining the required quantity $\Delta P$ from eq. 1 . This correction pertains the MDT on which the temperature is measured, but it could also be valid, at least, for the 5 neighboring MDTs connected at the same manifold. Actually, the observed temperature uniformity inside the box allows the application of the above correction to all the MDTs of the particular layer without a significant error.

An additional correction to the measured differential pressure must be taken into account, when the MDT is connected to the manifold. The reason is that the small volume of the manifold affects the actual pressure in the MDT. This correction, according again to the ideal gas law, is:

$$
\Delta P^{\prime}=(1+a) \Delta P^{\prime \prime}-a \Delta P_{m}^{\prime}=\Delta P^{\prime \prime}+a\left(\Delta P^{\prime \prime}-\Delta P_{m}^{\prime}\right)
$$

where, $\Delta P^{\prime \prime}$ is the differential pressure of the system tube + manifold, $\Delta P_{m}^{\prime}$ is the differential pressure of the manifold and $a$ is the ratio $V_{m} / V$, where $V, V_{m}$ is the volume of the MDT and the manifold, respectively.

A typical value of $V_{m}$ is $0.04 l$, which is almost the $1 / 30$ of the MDT volume, hence $a \cong 0.033$. The pressure in the manifold usually differs from that of the tested MDT pressure by less than 2 mbar. This corresponds to a term $a\left(\Delta P^{\prime \prime}-\Delta P_{m}^{\prime}\right) \approx 0.07$, which also leads to a relative correction of less than $2 \%$ with respect to the $\left(10^{-8}\right.$ bar $l / s$ ) nominal gas leak rate value. 


\subsection{Statistical error of the gas leak rate}

In the calculation of the pressure drop, eq. 14, the overall statistical error can be estimated as follows:

$$
\delta \Delta P=\sqrt{\left(\Delta P^{\prime} \frac{\delta T_{o t}}{T_{t}}\right)^{2}+\left(\frac{T_{o t}}{T_{t}} \delta \Delta P^{\prime}\right)^{2}+\left[\delta\left(P_{r} F_{T}\right)\right]^{2}}
$$

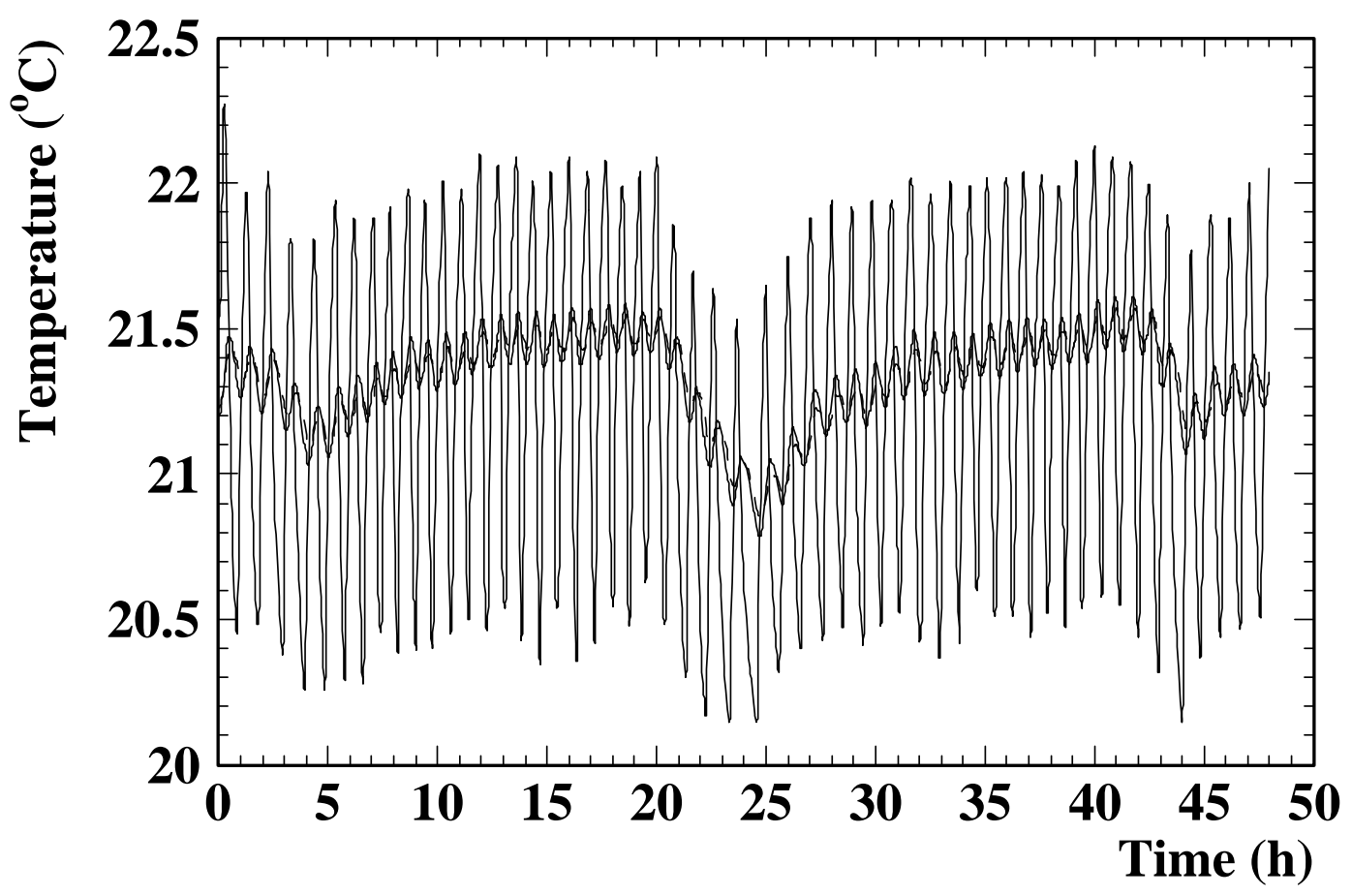

Figure 4. Temperature evolution with the time during the gas leak rate measurement inside one of the thermal stabilization boxes. The solid line curve corresponds to the MDTs and the dashed line corresponds to the reference tube temperature, respectively. The curve with the large variations, coming from the air conditioning cycles, is the room temperature. These variations are reduced sufficiently inside the box due to the thermal isolation.

The term $P_{r} F_{T}$ can be calculated from the known $F_{T}$, considering the pressure $P_{r} \cong$ 3 bars, while its variation can be recorded during the gas leak rate measurement. The statistical fluctuations of $F_{T}$ must be within an allowed practical range, i.e. $\pm 3 \times 10^{-4}$ maximum. As discussed above, the term $P_{r} F_{T}$ expresses a systematic error in measuring the pressure drop. Due to the finite time duration of the pressure recording, this term shows a certain variation. Thus, in the calculation of the overall error of $\Delta P$, from eq. 13, we consider a partial contribution of the variance of the term $P_{r} F_{T}$, that is $\left[\delta\left(P_{r} F_{T}\right)\right]^{2}$ $=\sigma_{T}^{2}$. Substituting this term in the eq. 17 we obtain:

$$
\delta \Delta P=\sqrt{\left(\Delta P^{\prime} \frac{\delta T_{o t}}{T_{t}}\right)^{2}+\left(\frac{T_{o t}}{T_{t}} \delta \Delta P^{\prime}\right)^{2}+\sigma_{T}{ }^{2}}
$$


The temperature evolution inside and outside the thermal stabilization box is presented, in figure 4 , for a typical test. It is found that, during the measurement, the factor $F_{T}$ varies in a maximum range from $\pm 1.5 \times 10^{-4}$, which corresponds to a variation of about $0.015 \%$ around the mean value.

It is has been calculated, that the range of the factor $F_{T}$ corresponds to a variation of the temperature difference, $T_{t}-T_{r}$, of about $0.05{ }^{\circ} \mathrm{C}$. The measured temperature variation at the MDTs array was systematically found to be less than $0.05{ }^{\circ} \mathrm{C}$ r.m.s., corresponding to values $F_{T}<1.5 \times 10^{-4}$ and $\sigma_{T}=0.45 \mathrm{mbar}$.
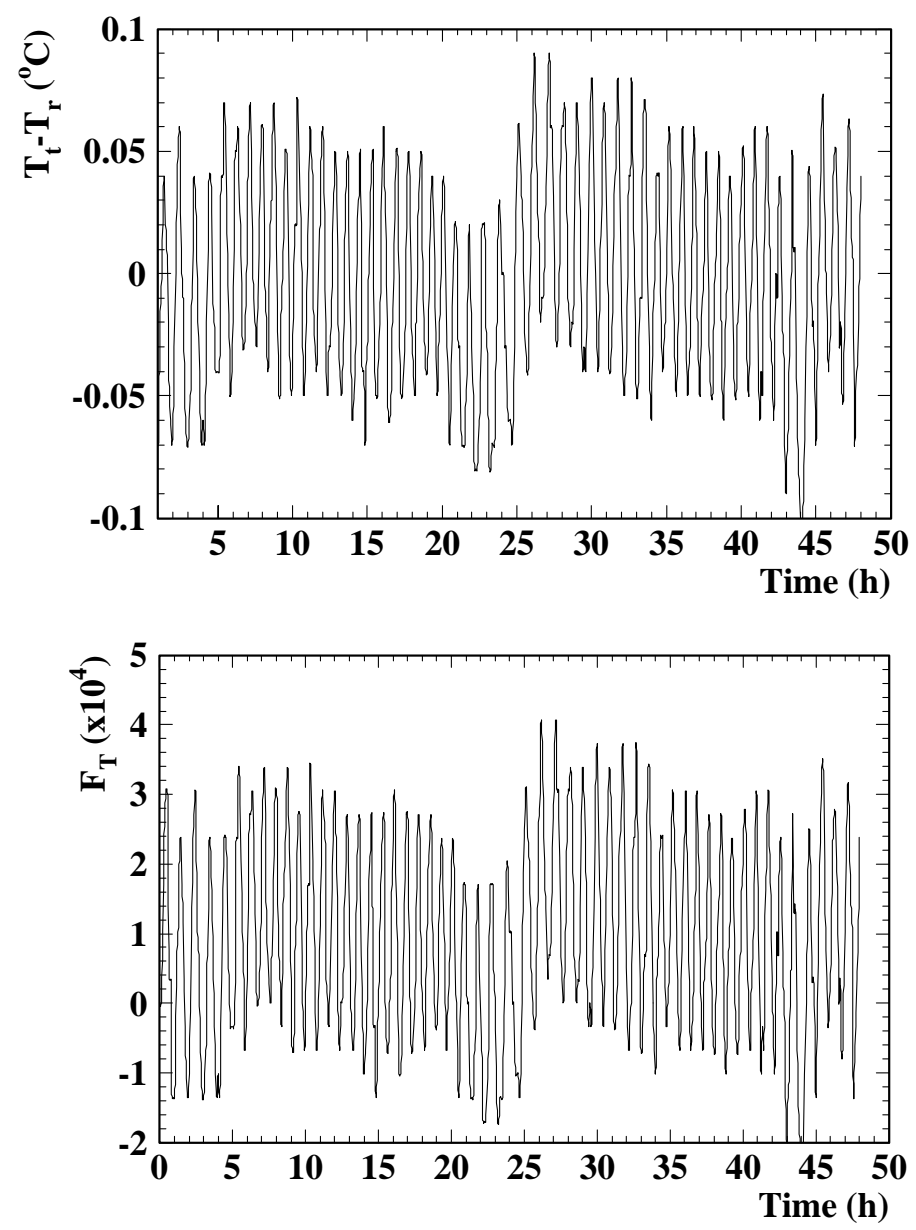

Figure 5. Temperature difference $\left(T_{t}-T_{r}\right)$ evolution and the Temperature Influence Factor, $F_{T}$, is shown in the upper and lower part of the plot, respectively. The two quantities reflect the same phenomenon.

The temperature difference $T_{t}-T_{r}$ and the factor $F_{T}$, are shown at the upper and lower part of the figure 5 respectively, where the r.m.s. variation is about $0.54 \times 10^{-4}$ corresponds to a temperature difference of $0.02{ }^{\circ} \mathrm{C}$.

The absolute error of the differential manometer is $0.1 \%$ in the full scale, that is $\delta \Delta P^{\prime}=0.35$ mbar. Also, due to the high accuracy of the thermometer $\left(\delta T=0.01{ }^{\circ} \mathrm{C}\right)$ and the low value of $\Delta P^{\prime}$ (e.g. 1.55 mbar for a time interval of $\left.48 \mathrm{~h}\right)$, the first term, $\left(\Delta P^{\prime} \delta T_{o t} /\right.$ $\left.T_{t}\right)^{2}$ of the eq. 18, is negligible compared to the other two terms.

Typical values of the squared terms, in eq. 18, are 2.7. $10^{-9} \mathrm{mbar}^{2}, 0.12 \mathrm{mbar}^{2}$ and $0.20 \mathrm{mbar}^{2}$ respectively, resulting to an absolute statistical error $\delta \Delta P^{\prime} \cong 0.56 \mathrm{mbar}$ and a 
relative one $\delta \Delta P / \Delta P^{\prime}=0.56 / 1.55=0.36$. Finally, the global statistical error of the gas leak rate $L$, is given by:

$$
\delta L=\frac{V}{\Delta t} \sqrt{\left(\frac{T_{o t}}{T_{t}} \delta \Delta P^{\prime}\right)^{2}+\sigma_{T}^{2}}
$$

A typical value is $\delta L \sim 0.06 \times 10^{-8} \mathrm{bar} \cdot \mathrm{l} / \mathrm{s}$, that is $\sim 6 \%$ with respect to the nominal leak rate. The main contribution to the error of $L$, comes mostly from the second term expressing the variation of the temperature difference.

\subsection{Compensation against the temperature variation}

Our system for the gas leak rate measurement has been extensively tested, using 6 MDTs, for 16 days, sampling the pressure drop of each MDT per day. The aim of this test is to study the influence of the temperature variations, for a long time period, on the leak rate. In fig. 6 the obtained results during the test are shown. The variations of the leak rate measurements by the time are correlated due to the common effect and reduced progressively. Consequently, for a time interval of 2-3 days, a temperature compensation is necessary to the obtained gas leak rate measurements during the MDT mass production.

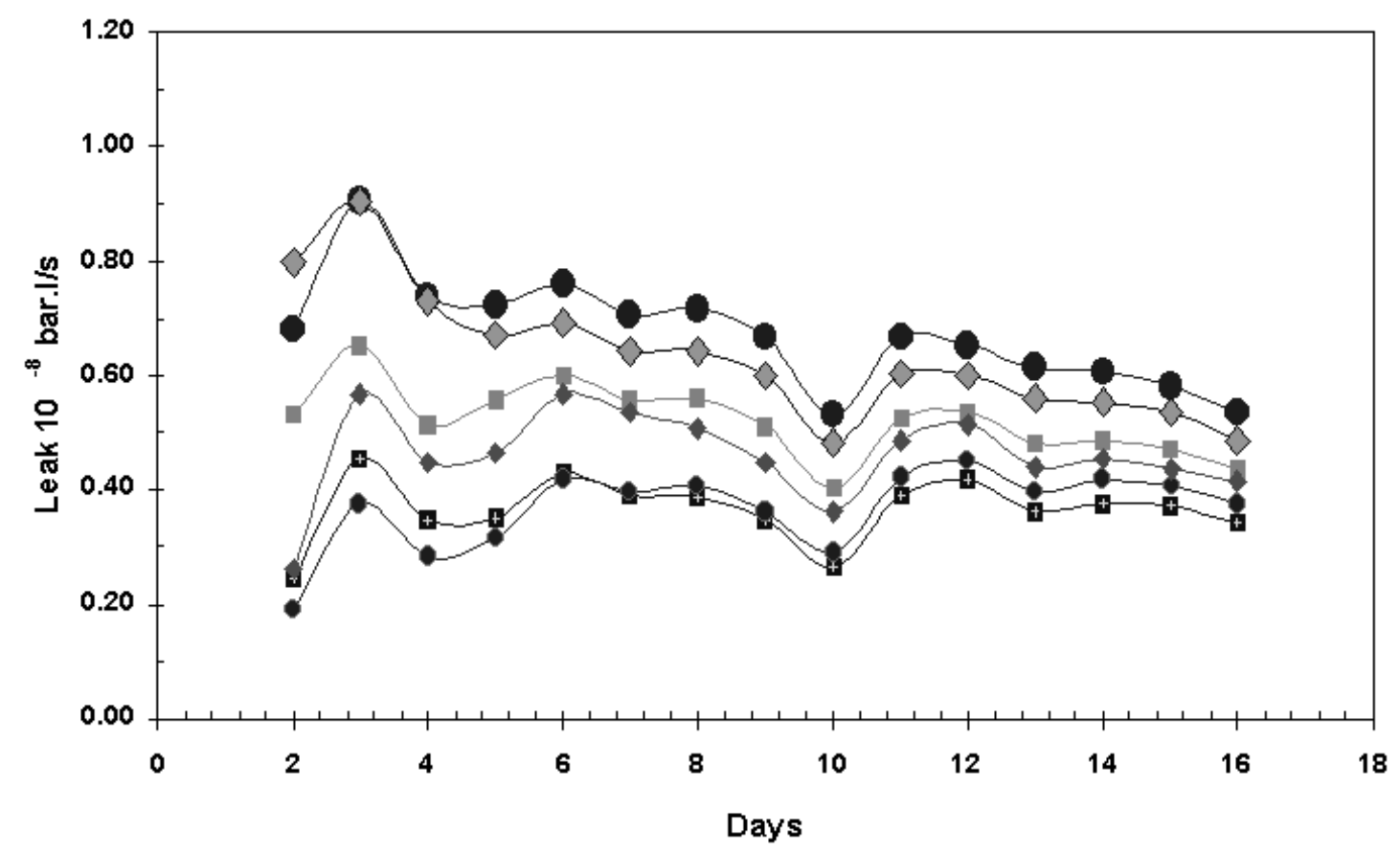

Figure 6. The monitored, for 16 days, gas leak rate measurement of several MDTs, is shown. The mean leak rate value for all MDTs, after 8 days monitoring and measurement, reflect the time stability and reliability of the setup.

In order to reduce the time interval of the gas leak rate test, i.e. to complete the measurement in a short time of $48 \mathrm{~h}$, the compensation of the systematic error, caused by the temperature effects, is quite indispensable. This compensation is obtained by calculating the term $P_{r} F_{T}$ according to eq. 15. In this correction, we have to assume that 
the temperature uniformity is maintained sufficiently along the particular MDT layer, even though some MDTs away from the vicinity of the temperature sensor are not corrected precisely. In addition, possible small temperature variations caused by slight air streams inside the TSB, usually do not transferred sufficiently inside the gas conditions of the tube. Therefore, we have to be careful performing such a correction using, initially, only two temperature sensors. For this reason, the temperature monitoring system was improved installing one sensor per group of 5 MDTs (1 per manifold), which increases the reliability of the compensation.

An additional "auto"-compensation technique has been implemented, which is based on using "calibration tubes" with a measured gas leak rate, obtained either by accurate measurement for a long time interval, tightened well in thermal contact with the MDTs under test, or taken via a mass spectrometer complex apparatus [8]. In that configuration, the temperature conditions inside the "calibration tubes" are similar to that of the MDT under the gas leak rate test.

Recent results of this study have shown that such a method leads to very reliable corrections. The compensation can be done in the off-line analysis with the following formula:

$$
\Delta P_{c}=\left(L_{m}-L_{o}\right) \frac{\Delta t}{V}
$$

where, $\Delta P_{c}$, is the compensation term, $L_{m}$ is the measured gas leak rate and $L_{o}$ is the well known gas leak rate, which is almost zero when using well tightened tubes. The resulted pressure drop $\Delta P$ is given by adding the compensation term, $\Delta P_{c}$ to $\Delta P^{\prime}$, so:

$$
\Delta P \cong \Delta P^{\prime}+\Delta P_{c}
$$

The experimentally measured values of $\Delta P_{c}$ are mostly within the range from \pm 0.45 to 0.45 mbar, depending on the temperature conditions. For the nominal gas leak rate tolerance $\left(1 \times 10^{-8}\right.$ bar $\left.\cdot l / s\right)$ of the BIS chamber, the corresponding pressure drop rate is $0.78 \mathrm{mbar} / d a y$, a value which could be affected strongly by the temperature effects during a day, leading to a significant systematic error. The above described compensation techniques are used simultaneously for cross checking in order to prevent erroneous acceptance or rejection of a MDT under test.

\subsection{Absolute gas leak rate calibration}

As discussed previously, the gas leak rate measurement is always referred to the reference tube, which is a stainless steel constructed and well-tightened tube of equal dimensions of the MDTs under test. The absolute gas leak rate value of the reference tube, although it is considered to be negligible, is nevertheless unknown. This quantity can be eliminated, by using capillary tubes (CT) with selected dimensions connected with any of the MDTs. This method is based on the fact, that we can predict the gas leak rate value of the CTs using the appropriate theoretical models and compare them to our experimental results. This technique consists the principle of the on-line auto-calibration system of our gas leak rate measurement setup. More technical details can be found in reference [9]. 
The gas leak rate measurements have been performed using two CT's with inner radius $5 \mu \mathrm{m}$ and lengths 350 and $111 \mathrm{~mm}$ respectively, connected either separately or together in a serial way. The gas leak rate measurement with the CT of total equivalent length of $461 \mathrm{~mm}$ was found to give a value $(1.063 \pm 0.020) \times 10^{-8} \mathrm{bar} \cdot \mathrm{l} / \mathrm{s}$, which is close to the ATLAS muon spectrometer standard for the MDTs. This result was obtained for a total time interval of several days. The theoretical expected value is $1.025 \times 10^{-8} \mathrm{bar} \cdot \mathrm{l} / \mathrm{s}$, therefore, the two values, measured and calculated are consistent. It should be taken into account that the radius of the CT has been determined experimentally, by obtaining the cross section image of the capillary with an optical microscope and compared to that of an optical grating with 300 lines $/ \mathrm{mm}$ corresponding to $3.33 \mu \mathrm{m}$ per line.

\section{Experimental results}

\subsection{Acceptance criteria}

The ATLAS muon spectrometer has a performance upper limit for the gas leak rate of each MDT of $L_{u p}<1 \times 10^{-8} \mathrm{bar} \cdot \mathrm{l} / \mathrm{s}$.

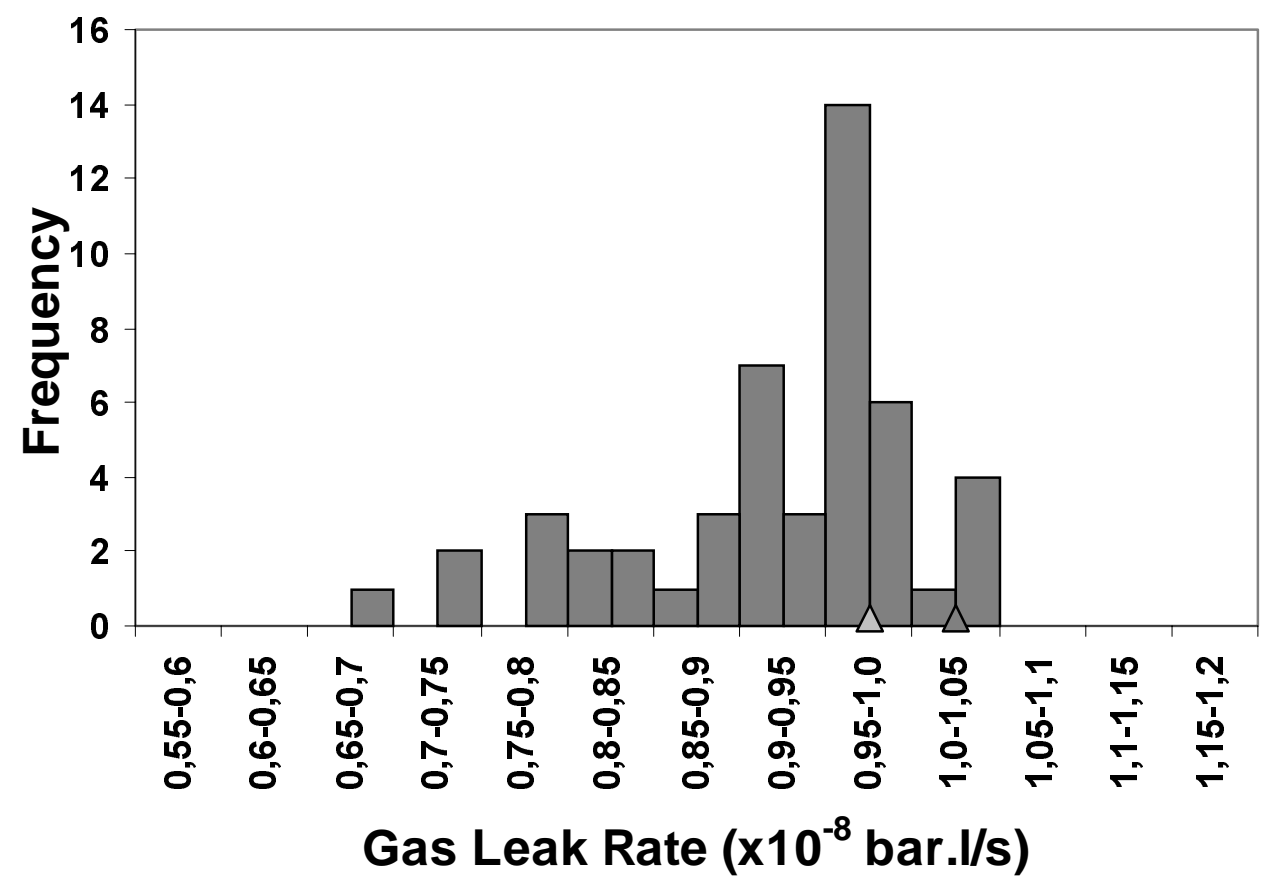

Figure 7. A bar graph showing the obtained gas leak rate distribution from a typical gas leak rate measurement. The light color bars represent the distribution resulting from the MDTs lower layer, while the dark color bars the distribution from the MDTs upper layer. The light and dark arrows show the position of the modified upper limits respectively, that is $1.01 \times 10^{-8} \mathrm{bar} \mathbf{x} \mathrm{l} / \mathrm{s}$ and $1.04 \times 10^{-8} \mathrm{bar} \mathbf{x} \mathrm{l} / \mathrm{s}$. 
Due to the finite range of the distribution of the gas leak rate obtained by our developed setup and the systematic errors, the criteria for accepting every tested MDT must be well specified. A sequence of steps is described below based on these criteria.

The statistical fluctuations of the MDT production are expressed by the standard deviation, $\sigma_{p}$, which is in principle, an unknown quantity. In paragraph 3.3, the statistical error of our measurement, $\sigma_{s}$, it is given (eq. 5). Consider that it follows a Gaussian probability distribution, $g_{s}$. The estimated value of $\sigma_{s}$ is $0.05 \times 10^{-8} \mathrm{bar} x \mathrm{l} / \mathrm{s}$. In addition, the standard deviation of the gas leak rate, $\sigma_{t}$, obtained by testing a group of 25 tubes is $0.08 \times 10^{-8} \mathrm{bar} x \mathrm{l} / \mathrm{s}$, assuming also a Gaussian distribution $g_{t}$.

The average value of the gas leak rate, $L_{t}$, is expected to be varied from time to time, due to systematic effects, while the standard deviation is expected to be maintained within a narrow range because it is specified by the methods of MDTs production and the gas leak test. This fact has been confirmed by our measurements.

The convolution of $g_{p}$ taking into account, $g_{s}$, causes a broadening to the first according to the process $g_{t}=g_{p} * g_{s}$. Consequently, the unknown $\sigma_{p}$ is given by:

$$
\sigma_{p}=\sqrt{\sigma_{t}^{2}-\sigma_{s}^{2}}
$$

Using the typical previously given arithmetic values, we obtain the error value $\sigma_{p}$ $=0.06 \times 10^{-8} \mathrm{barxl} / \mathrm{s}$.

In the following, $L_{t}$ is the mean value of the gas leak rate values of 25 MDTs obtained from our setup. The aim is to make a decision if a particular MDT should be accepted satisfying the performance of the upper limit $L_{u p}$.

The appropriate rejection criteria are described, containing the following steps:

i. Calculate the mean $L_{t}$ and the standard deviation $\sigma_{t}$ separately for each layer, from the obtained gas leak rate measurements.

ii. Considering the distribution $g_{p}$, calculate the quantity $\sigma_{p}$ from eq. 22 .

iii. Find the modified upper limit for both layers, $L_{\text {upm }}$ based on the condition:

$$
P\left(Z \leq\left(L_{u p}-L_{t}\right) / \sigma_{p}\right)=P\left(Z \leq\left(L_{u p m}-L_{t}\right) / \sigma_{t}\right)
$$

where, $Z$ is the variable of the normal distribution. The equality means that we claim to have equal probability for the gas leak rate of an MDT to be below the limits in both probability distributions $g_{p}$ and $g_{t}$, respectively. This leads to the result:

$$
L_{u p m}=L_{t}+\left(\sigma_{t} / \sigma_{p}\right) \cdot\left(L_{u p}-L_{t}\right)
$$

applying the typical values mentioned above we obtain the value: $L_{u p m}=1.33 \times 10^{-8}$ barxl/s.

iv. Accept the MDT if $L_{t}<L_{u p m}$ within an uncertainty $\delta L_{u p m}$ expressed by the error $\delta L_{t}$. This error comes from the imperfect compensation of the temperature effects. Assuming $\delta \sigma_{t}$ and $\delta \sigma_{p}$ to be much smaller than $\delta L_{t}$, the corresponding error $\delta L_{u p m}$ is given by: 


$$
\delta L_{u p m} \cong\left|\left(1-\frac{\sigma_{t}}{\sigma_{p}}\right) \delta L_{t}\right|
$$

v. Calculate the probability to accept an MDT within this uncertainty by integration of the normalized probability distribution $g_{t}$ from the limit $L_{l}=L_{u p m}-\delta L_{u p m}$ to $L_{2}=L_{\text {upm }}$, as follows:

$$
P_{\text {up }}=P\left(L_{1} \leq L_{i} \leq L_{2}\right)=\int_{L_{1}}^{L_{2}} g_{t}(L) d L
$$

For a given upper bound $a$ of this probability, where $a=0.05$ or $5 \%$, check if $P_{u p}<a$ to verify the validation of the step (iv).

\subsection{Evaluation measurements}

The gas leak rate measurement of more than 6000 MDTs with various possible was evaluated with the present setup. The presented results, fig. 8 , are referred to the obtained mean value for every 25 MDTs per layer in the box.

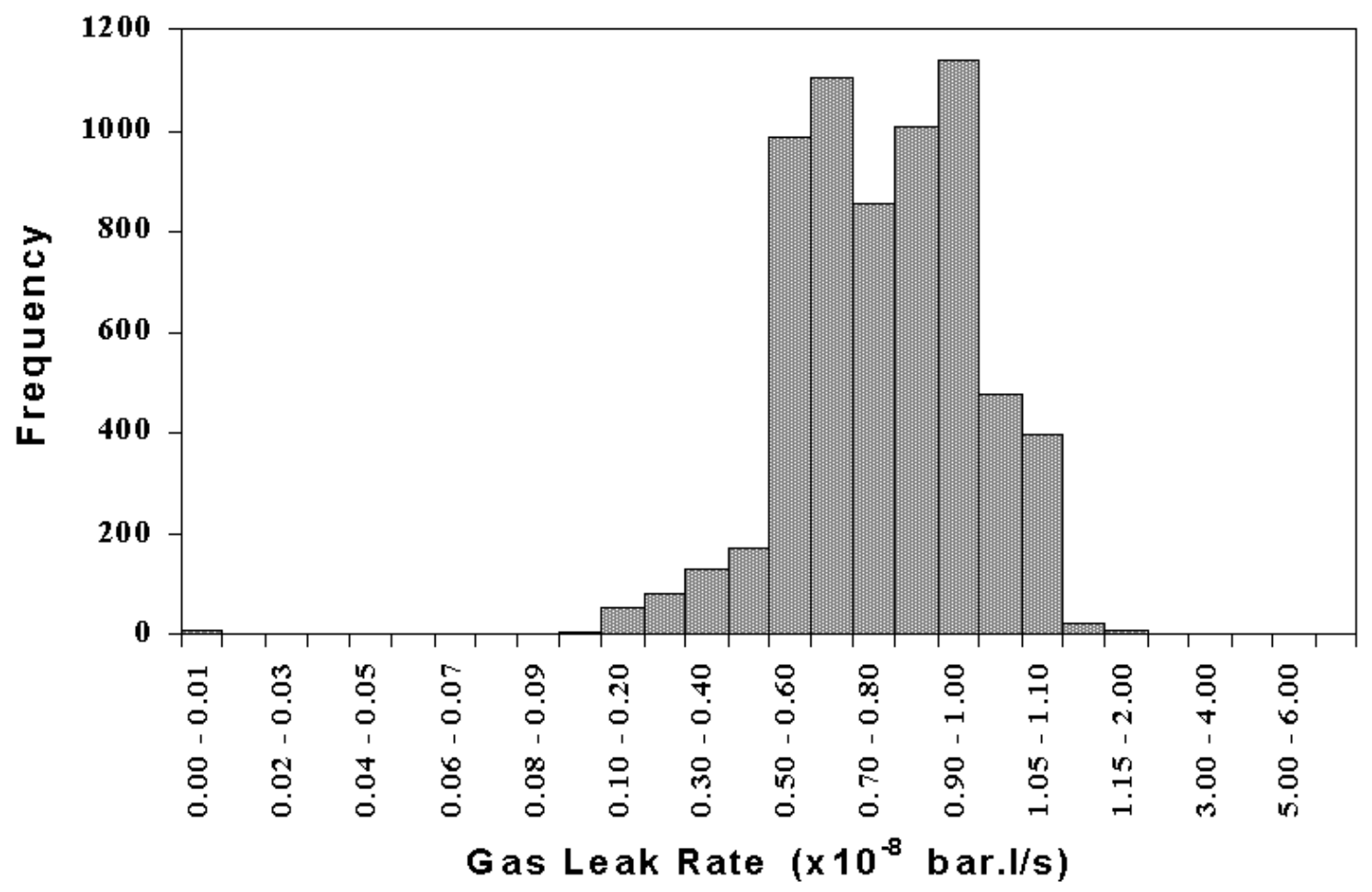

Figure 8. The gas leak rate measurement distribution for more than 6000 tested MDTs. 
The obtained mean standard deviation of the gas leak rate measurement, is $\sigma_{t} \cong$ $0.08 \times 10^{-8} \mathrm{barxl} / \mathrm{s}$. This value includes the statistical fluctuations of both the measurement and the production, as well. The latter are due to the variation of the material quality and the construction parameters (eg. the crimping force between endplug and cylinder).

Furthermore, the covariance of the gas leak rate value has been studied by performing two successive measurements of the same MDTs, separated by a $48 \mathrm{~h}$ time interval. A linear correlation coefficient $\rho=0.85$ was found between the two measurements, leading to the conclusion that the statistical uncertainties are sufficiently small.

The measured gas leak rate distribution result from a typical MDT gas leak measurement is shown in fig. 8. The modified upper limits have been calculated according to the discussed algorithm. In this particular test, two tubes were rejected: one tube because of its large gas leak rate due to the fact that the O-ring of one of the endplugs was not in its appropriate position and the second one due to a slightly higher gas leak rate value of the permitted upper limit. The first tube was re-measured passed the acceptance criteria.

\section{Conclusions}

We have developed and operated a gas leak rate measurement setup for the QA_QC mass production test of the MDTs based on the determination of the pressure drop within a certain time interval. After testing several thousands of tubes, the performance of the system is found to be appropriate for performing the gas leak rate QA_QC of the MDTs with the expected reliability. The sensitivity in the temperature variations has been faced using monitoring system and auto-compensation techniques. An absolute calibration method of the system has been done using capillary tubes. Satisfactory consistency between theoretical models and the specific experimental results have been found.

\section{Acknowledgements}

This work has been performed within the ATLAS Collaboration, and we thank the several collaboration members for helpful discussions, we have made use of information and ideas, which are the result of collaboration-wide efforts. We thank Dr. H. Wellenstein (Brandeis University) for helpful discussions and providing us with some technical material. We, also, thank Prof. Dr. W. Blum (MPI-CERN) for his continuous interesting and encouragement to our effort on the project. We also thank our colleagues Drs. A. Maltezos, N. Papadakis and N. Vodinas for their help to the measurements and the technical staff, of our department, for their contribution to the system construction.

This work has been partially supported by the Greek Ministry of Development, the General Secretariat of Research \& Technology, projects 98 MIK-09 and 98 MIK-21. 


\section{References}

[1] ATLAS Collaboration, CERN/LHCC/97-22, Muon-TDR 10, Technical Design Report (1997).

[2] D. Fassouliotis et al., MDT BIS module-0 tube assembly, ATL-MUON-2000014, (ATL-COM-MUON-99-031).

[3] M. Dris, et al., The QA_QC Results of the BIS-Module-0 Monitored Drift Tubes, ATL-MUON-2000-019, (ATL-COM-MUON-99-036 ).

[4] Sampsonidis D. et al., Construction of the BIS MDT Chamber Module-0, ATLCOM-MUON-2001-006.

[5] G Chelkov et al., Differential Manometer for gas leak measurement, http://atlas.web.cern.ch/Atlas/project/MDT/www/mirrordubna/atlas/QAQC/chle ak/dfmanom.htm

[6] Th. Alexopoulos, et al. The Quality Assurance_Quality Control of the Monitored Drift Tubes at the HEP Laboratory of the National Technical University of Athens, ATL-MUON-2001-017.

[7] H. Burckhart, Local Monitor Box (LMB) for the ATLAS-DCS, http://atlasinfo.cern.ch/ATLAS/GROUPS/DAQTRIG/DCS/lmb.html

[8] Th. Alexopoulos, et al., A low cost accurate system for multi-point temperature measurement and processing, in preparation for ATLAS Note.

[9] W. Blum, Gas leaks in the insulator material of the endplugs, ATLAS Muon status report, 20 August 1999, http://blum.home.cern.ch/blum/Norylcracks.html

[10] V.T. Braic, et al., Leak detection system for mass-production of DT detectors, ATL-MUON-1998-225.

[11] S. Maltezos, et al., Calibration of the NTU-Gas Leak Rate Measurement System using Capillary Tubes, in preparation for an ATLAS Note. 